\title{
Le défibrillateur au cabinet dentaire
}

\author{
Florian Laurent ${ }^{1}$, Pascal Augustin ${ }^{3}$, Cathia Zak ${ }^{1}$, Louis Maman ${ }^{1}$, Nicolas Segal ${ }^{2}$ \\ ${ }^{1}$ Université Paris Descartes, Service d'Odontologie, Hôpital Charles Foix, 7 avenue de la République, \\ 94200, Ivry sur Seine, France \\ ${ }^{2}$ Service d'Accueil des Urgences, Hôpital Lariboisière, 2 rue Ambroise Paré, 75010 Paris, France \\ ${ }^{3}$ Réanimation chirurgicale, Hôpital Bichat Claude Bernard, 46 rue Henri Huchard, 75018 Paris, \\ France
}

\section{florian.laurent@free.fr}

Introduction: La défibrillation précoce est l'un des maillons important de la chaine de survie lors d'un arrêt cardiaque. Même si son incidence est faible, le chirurgien-dentiste peut y être confronté au cabinet dentaire (Calon et al. 2009). L'objectif de cette étude était d'évaluer l'équipement en défibrillateur des cabinets dentaires, la prévision d'équipement dans l'année qui suivait et les raisons de non équipement.

Matériel et Méthodes: Entre octobre et décembre 2010, nous avons mené une étude téléphonique prospective en appelant tous les cabinets dentaires omnipraticiens de la ville de Paris (France).

Résultats: Les 1624 cabinets dentaires omnipraticiens de Paris ont été appelés: 1287 (79\%) ont été contactés et $24(1.9 \%)$ ont refusé de répondre. Seulement 33 cabinets $(2.6 \%)$ étaient équipés d'un défibrillateur: 10 (31\%) d'un défibrillateur automatique, 14 (42\%) d'un semi-automatique et $9(27 \%)$ ne connaissaient pas le type de leur défibrillateur.

94.5\% des cabinets dentaires non équipés ne prévoyaient pas d'acquérir un défibrillateur dans les douze mois suivants. Les principales raisons données pour expliquer le non équipement étaient : le sentiment d'inutilité d'un défibrillateur en cabinet dentaire $(24.8 \%)$, le prix trop élevé $(18.5 \%)$, l'absence de besoins antérieurs (10.7\%), l'absence d'obligation d'avoir un défibrillateur en cabinet dentaire $(6.9 \%)$ et l'approche de la retraite $(6.4 \%)$.

Discussion: Cette étude a montré le sous-équipement des chirurgiens-dentistes en défibrillateur. Par ailleurs, elle révèle que cette catégorie de professionnels de santé ne souhaite pas s'équiper à court terme. Au vu des raisons invoquées, leur réticence à s'équiper semble difficilement surmontable. Il parait donc nécessaire de trouver une solution alternative afin que la défibrillation soit disponible au cabinet dentaire.

Sachant que l'European Resuscitation Council recommande l'équipement des zones résidentielles en défibrillateur et que la quasi-totalité des cabinets dentaires se trouve dans des bâtiments d'habitation, il pourrait être envisagé un investissement commun et la mutualisation d'un défibrillateur à l'échelle d'un même bâtiment, avec les résidents et les autres professionnels de santé : cabinets médicaux et pharmacies.

Conclusions: Cette étude a montré que malgré les recommandations scientifiques actuelles, les cabinets dentaires français sont sous-équipés en défibrillateur. La possibilité de partager le coût de l'équipement entre plusieurs professionnels de santé d'un même immeuble pourrait être une solution. 